\title{
The Lived Experiences of Chinese International College Students and Scholars during the Initial COVID-19 Quarantine Period in the United States
}

\author{
Yaoying $\mathrm{Xu}^{1}{ }^{\mathrm{D}} \cdot$ Donna Gibson $^{1} \cdot$ Toshna Pandey $^{1} \cdot$ Yingying Jiang $^{1}$. \\ Benjamin Olsoe ${ }^{1}$
}

Accepted: 9 July 2021 / Published online: 28 July 2021

(c) The Author(s), under exclusive licence to Springer Science+Business Media, LLC, part of Springer Nature 2021

\begin{abstract}
The purpose of this study was to explore Chinese international college students' lived experiences during the initial COVID-19 quarantine period in the United States. Using a descriptive phenomenological psychological method, data were gathered from 14 international students and visiting scholars from China using individual and focus group interviews. Four transformed meaning units that formed the structural base of the phenomenal experience were identified as Safety Concerns, The Salience of the English Language, Intersectionality of Policies and Complex Decision Making, and Unexpected Support and Benefits. Implications for policy making and support for international students' learning in the U.S. higher education institutions and future research recommendations are included.
\end{abstract}

Keywords COVID-19 · Chinese international students · Lived experiences

\section{Introduction}

The United States has hosted the largest share of international students globally, with more than one million each year for five consecutive years (Open Doors Report, 2020). Among all international students in the U.S., Asian students are the majority, with the largest group from Mainland China (35\%) (Open Doors Report, 2020). In 2020, about 400,000 Chinese students were studying in the U.S. (Amour, 2020). Even with an overall decline in enrollment of international students since the pandemic, Chinese students continue to represent the largest portion of all international students in the U.S. (Open Doors Report, 2020).

Yaoying Xu

yxu2@vcu.edu

1 Virginia Commonwealth University, 1015 W. Main St., P.O. Box 842020, Richmond, VA, USA 
Undoubtedly, COVID-19 has impacted everyone's life globally. As a group, college students seem to experience more challenges during the pandemic due to the unique transition time from being at home to being independent individuals away from home. The lack of meaningful social interaction has created additional challenges to this group of young people (Bilecen, 2020). Compared to domestic college students, international students need more social interaction in order to adapt to the new culture which would be expected as part of their routine experience until the onset of the pandemic. The outbreak of COVID-19 affected all international students due to the interruption of academic work, the disconnect with the community, the inability to travel back to their home countries as a result of closed borders and suspended international flights which caused stress and anxiety (Bilecen, 2020), and restrictions of immigration policies. The challenges for Chinese international students have been tremendous and multifaceted under the unique sociocultural and political circumstances between China and the U.S. (Ma \& Miller, 2020). As Tavernise and Oppel (2020) noted in the New York Times, "ChineseAmericans face a double threat. Not only are they grappling like everyone else with how to avoid the virus itself, they are also contending with growing racism in the form of verbal and physical attacks" (para. 4). In some instances, Asian individuals in the U.S. encountered racist attacks for wearing masks (Palmer, 2020) as well as not wearing them (Henry \& Bensimon, 2020).

The COVID-19 pandemic affects international student mobility in general, although the extent varies due to sociocultural, economical, and political complications for different groups of international students. Researchers and educators have taken quick actions to examine the impact of the pandemic on Chinese college students or international Chinese college students' educational outcomes and mental health during the COVID-19 pandemic. For example, Chi et al. (2020) examined the prevalence and risk factors for poor mental health of Chinese university students by conducting a nationwide online survey of over 2000 students in China and found that over 30\% of respondents indicated symptoms of posttraumatic stress disorder. Yu et al. (2021) did a similar survey study on Chinese college students and their results were even more alarming with almost $57 \%$ of respondents $(n=1681)$ indicating depressive symptoms. Compared to these students in China, international Chinese students experienced more challenges that could impact their mental health. Using web-based research, Wang (2020) demonstrated different stressful situations Chinese international students in the U. S. experienced at the early and later stages of COVID-19. In the early stage when the virus was first discovered in China, Chinese international students received criticism for "bat eating", stocking up facial masks to send back home, and the Wuhan lockdown. In the later stage, when the outbreak of COVID-19 started in the U.S., Chinese international students received contradicting messages. Families and friends urged them to go back to China (when China had stabilized the situation). However, infrequent flights between the two countries implied that students should stay in the U.S. to avoid the spread of the virus in both countries (Ma \& Miller, 2020). Other predicaments of policy changes included the possibility of being unable to return to the U.S. due to the travel ban imposed by the U.S. government and the threat of being forcefully sent back to their home countries in the absence of in-person class enrollment. As Wang (2020) indicated, "This group of international students is the beneficiary of 
globalization and international education exchange yet witnessing the trend of anti-globalization and nationalism (p. 2)"'.

Within a year a number of rapid studies that have been conducted to investigate Chinese college students' mental health during COVID-19 (e.g., Chi et al., 2020; Yu et al., 2021) or Chinese international students' wellbeing during the pandemic (e.g., Roberto et al., 2020; Schild et al., 2020; Wang, 2020). Most of the studies took advantage of the efficiency and convenience of wide spreading social media or web-based sources through digital surveys to identify the overall trends. Both $\mathrm{Chi}$ et al. and $\mathrm{Yu}$ et al. designed cross-sectional survey studies to reach out a large number of respondents using a variety of social media tools such as Wechat or Weibo. These survey studies provide very useful information on Chinese students or international Chinese students' overall wellbeing as a group and demographic characteristics to help illustrate their educational background. However, very few studies have examined Chinese international students' experiences closely using in-depth data collection and analysis beyond web-based questionnaires. The purpose of this study was to explore Chinese international students' lived experiences in the age of COVID-19. Specifically, we intended to explore the intersectionality of these students' educational experience and culture within the pandemic context. The following research question was addressed: What are the lived experiences of Chinese international students during COVID-19?

\section{Method}

Because the researchers' intent was to examine the lived experiences of Chinese international college students during the initial COVID-19 quarantine period at a U.S.based university, a phenomenological qualitative method was chosen. The COVID-19 pandemic and subsequent required quarantine presented an international phenomenon that has not been experienced in over a century. Due to the origins of the virus, Chinese international students and scholars located at U.S. higher education institutions were susceptible to prejudice and discrimination. In order to better understand their lived experiences, a research method that encouraged them to share those experiences in depth and provide insight into the psychological meaning associated with those experiences was appropriate (Downing \& Cooney, 2012; Holloway \& Todres, 2003). More specifically, the descriptive phenomenological psychological method (Giorgi et al., 2017) allowed researchers to examine the psychological phenomenon described through the participants' descriptions of their experiences. The personal, psychological meaning conveyed through this process provided a more accurate and structured description of their experiences during this initial quarantine.

\section{Role of Researchers}

Consistent with descriptive phenomenological approaches, we strived to be transparent by disclosing our positionality in conducting this study as well as the potential influence it had on our writing. A total of five researchers were involved in this study and all are authors on this manuscript. Four of the five authors identify as 
female and the fifth author identifies as male. The first two authors have an average of 21 years of experience as university professors holding doctoral degrees in their respective professional fields. Two of the authors identify as doctoral students and one author is a program coordinator for an international studies center with all of them holding master degrees. Four of the five authors have experiences as international students, and three of the five authors are United States citizens. Researchers have an average age of 38-years-old, representing Chinese, Indian, and White ethnicities. The researchers were sensitive to potential additional challenges facing Chinese international students either due to their own experiences or through those of their students. In order to focus on the participants' experiences as unbiased as possible, we acknowledged our prior knowledge of international student experiences and bracketed our beliefs, values and assumptions when constructing the study and prior to interviews (Moustakas, 1994). Inherent in a descriptive phenomenological method, bracketing through memoing and discussion with fellow researchers and employing multiple methods to mediate biases and ensure the validity of the process throughout the study, as outlined in the data analysis section, allowed us to capture the lived experiences of Chinese international students more accurately.

\section{Participants and Procedures}

Due to the international COVID-19 pandemic, the authors' university called for special research proposals to examine multiple aspects of the effects of the pandemic. Consequently, this study proposal was selected for funding through this internal call for research. For this study, IRB approval was gained through the authors' institution of higher education. Because we sought to understand the lived experiences of Chinese International college students during the initial COVID-19 quarantine period in U.S.-based universities, we defined the sample as those who identified themselves as Chinese international students attending college in either undergraduate or graduate college programs during the 2020 spring academic semester (mid-January through mid-May). Additionally, two visiting scholars from China, one with a Ph.D. and another with a Master's degree, participated in the study.

Sampling occurred in four different ways. First, we contacted our university's global education office to invite all international students to participate via an email listserv. Second, students in the Chinese Students and Scholars Association (university student group) were sent the invitation to participate through their group listserv. Third, Chinese international students were contacted via WeChat with the invitation to participate. Fourth, authors contacted Chinese International students via email to invite peers (i.e., snowball) to participate with details being provided in an email invitation. The email invited potential participants to contact the authors to begin the process of consenting to and scheduling interviews. Utilizing these efforts at recruitment, 21 international students contacted us regarding the study. Five students were not included because they were not Chinese nationals. Two participants began the study but discontinued their participation due to different reasons. Therefore, a total of 12 students and two visiting scholars completed the study. A demographic questionnaire was sent to the participants through a SurveyMonkey link. At the time of 
the study, the average age of the participants was 30 years old. Thirteen out of the 14 participants identified themselves as female and one participant identified as male. In regard to their relationship status, 6 of the participants identified as single, 4 identified as married, and 4 identified as being in a relationship with a significant other.

In terms of their immigration status, 12 reported holding an F1 visa and 2 holding a J1 visa. Six of them identified as graduate students, 4 identified as undergraduate students, and 4 as either visiting scholars or students in English language proficiency (ELP) programs until status changed. At the time of the interviews (May and June, 2020), the majority (11 out of 14) of the participants were still residing in the United States.

\section{Data Collection}

We collected data virtually via Zoom using semi-structured interviews with a list of questions to guide the interview process and follow up questions for clarifications. We collected data using individual interviews and focus groups. The intention of the interviews was to elicit an in-depth exploration of their experiences since the advent of quarantine resulting from the COVID-19 pandemic in the U.S. Consistent with the structure of an intensive interview, the questions ranged from closed-ended questions about their decision to attend the university (e.g., Can you tell us why you chose $\mathrm{ABC}$ university?), open-ended broad questions about their experiences after quarantine (e.g., Tell us about your experience as international students since Jan 2020?), and more specific questions related to factors that could impact their experience (e.g., We would like to hear about your experience using social media as communication and social interaction. Tell us about your experience ...if you could give some examples, that would be helpful?). These questions were developed from recent literature on the potential impact of the pandemic on Chinese individuals living in the U.S. (Litam, 2020), and the experiences of the first and fourth authors. Feedback on clarity was sought from the Chinese international student prior to use. All interested participants were asked about their availability to participate in a group discussion but were offered an alternative individual interview in the case of their unavailability at the agreed upon time by majority participants. Of the 14 consented participants, two indicated their inability to meet with the larger group, and therefore, participated in individual interviews. The meetings started with the research team introducing themselves and their roles in the study. Next, the participants were informed about the purpose and rationale of the study, and subsequently, the research team answered questions and addressed concerns. The participants were informed that the session would be recorded, however, they were offered the choice to turn off their video to maintain anonymity. Next, they were also asked to change their display name on the screen to protect their identities. At this point, a key consisting of names and pseudonyms was created which was stored on a secure university web server. Finally, participants were reminded about their rights and were informed that their participation was voluntary.

The participants were informed that there would be one main facilitator/ interviewer and that the other members of the research team could ask follow-up 
questions, should they arise. After each question was asked, the interviewer called out individual participants to share their responses; the other participants were free to respond to others' responses. On average, each of the two interviews lasted an hour and each of the group discussions lasted approximately two hours. The facilitator closely monitored the participation, and encouraged the less vocal participants to share their experiences. At the end, the participants were thanked for their time and were alerted about a follow-up email with findings requesting their cooperation for member checking to ensure accuracy of data representation.

\section{Data Analysis and Trustworthiness}

The name key and the audio and video recordings of the interviews and group discussions were uploaded to a secure university web server to ensure participant anonymity and confidentiality. All recordings were transcribed verbatim using a university approved secure web transcription tool and saved to the secure web server which was only accessible to the research team.

The team used a Descriptive Phenomenological Psychological method (Giorgi et al., 2017) to analyze the raw data. The analysis began with four members of the research team reading all the transcripts individually to familiarize themselves with the essence of the described situations, followed by engaging in coding raw data, first as individuals and then as a team. Each member broke down larger sections into smaller units for analysis to delineate meanings from the unified description. This was done with the intention to distinguish parts with different meanings through careful rereading of the transcripts. Once all the meaning units were identified, they were transformed into phenomenologically and psychologically sensitive ways. This was done to transform the original expressions of the participants into their psychological meanings. Finally, using the transformed meaning units, the research team described the psychological expressions of participants' lived experiences (Giorgi et al., 2017).

In order to present evidence of authenticity, truthfulness, and credibility, we maximized the trustworthiness of our findings and conclusions by adhering to strong validity checks. This section will discuss the several trustworthiness criteria involved in this qualitative inquiry. During each step of the data collection and analysis phase, the research team sought to maintain the credibility and trustworthiness of findings. First, the team included data collection triangulation. For example, to test the validity of findings, the team converged multiple sources of data collection such as the interviews, data from demographic sheets, and responses from the member check surveys. A second form of triangulation, researcher triangulation (Lincoln \& Guba, 1985), was also included in this study. Specifically, each transcript was coded independently by four team members, after which these were discussed as a group. Next, to establish credibility, the researchers also ensured prolonged engagement with the participants by collecting demographic information and requesting member checks a few days after the interviews were conducted. Finally, we established the "believability" of our study and findings (Lincoln \& Guba, 1985) by providing rich 
descriptions of participant experiences, and identified and described participants' and the research team's characteristics accurately (Elo et al., 2014).

To ensure that the research process was accurate and transparent, the team made a consensus that all processes were logical, traceable, and clearly documented (Tobin $\&$ Begley, 2004). This was done to provide evidence for the decisions made regarding the theoretical and methodological issues throughout the research process. For example, all records of raw data, including audio and video recordings, transcripts, name keys, field notes, and responses from bracketing were stored on the secure web server.

\section{Results}

As described in the Method and Data Analysis sections, using the Descriptive Phenomenological Psychological Method to approach this research question required the researchers to discern meaning units from the raw data collected. These transformed meaning units formed the structural base of the phenomenal experience of Chinese International college students during the initial COVID-19 quarantine period at a United States-based university. For the participants, the experience produced unanticipated worries due to government enacted policies, lack of adherence to safety protocols, and social isolation. These factors were aspects of complex decision making around education, travel, and family responsibilities. The salience of gaining more confidence in the use of the English language through formal and informal means was highlighted and how expectations around utilizing English were not realized. Finally, participants experienced unexpected support and benefits through this experience that provided opportunities to feel connected to peers, professors, and community members. The evidence of the participant voices (all pseudonyms) of the four main transformed meaning units of Safety Concerns, The Salience of the English Language, Intersectionality of Policies and Complex Decision Making, and Unexpected Support and Benefits are included below.

\section{Safety Concerns}

Safety concerns were an overarching structure of participants' experiences. Initially, participants reported more concerns about families in China but this quickly transitioned to concerns about their own well-being while living in the United States. Emma reported,

I would say, like my cousins and aunts and uncles and such have been much more concerned about my residence in the US because of what they see on the news, they now message me every day so it's sort of a role reversal whereas in Dec/Jan I was reaching out to them, asking them if they were okay with the coronavirus situation and whether or not they were doing well. 
Managing their China-based family and friends' concerns about their safety and negotiating how to utilize safety measures in a society where classmates, roommates, and community members were inconsistent in adhering to safety measures were prevalent experiences. Nancy shared her experiences with friends in America:

We heard a lot of information from China and they treat us really seriously, like everyone using masks. But like in America, they don't really use masks like at the beginning. Now, I think, everyone is using masks. So, it's fine. Now when we go out, we're still using masks. But I don't see my friends really do that.

Due to inconsistent guidance from the government and university officials, safety protocols and how to address any illnesses left participants in potential hazardous situations. Mary describes an attempt to help her roommate get tested:

I actually have a friend and was very sick before and we had to call the doctor and no one wanted to see him and he cannot get a test for COVID-19, that was disappointing. Even a lot of people that support us and then want to get my friend tested but we did not get a test. I also have a lot of concerns about safety and health here.

Similarly, Catherine described the experience of her roommate becoming ill and their attempts at seeking help:

I think since April, my roommate started feeling, uh, uncomfortable in that, we are not sure why that is. And, um, she called the CDC, but they, um, they just responded that she's not qualified to do the test since she doesn't have the symptoms of all having difficulty breathing. So she only has a, I think coughing, sneezing, and also a little bit feverish, so all we are not sure what that is, but after she talk to the CDC, they didn't, they did just answer, she was not qualified to do the test, so she should do not do the test.

All of these experiences have fed concerns and possible anxiety about either remaining at the university or going back (some participants went home to China in the spring) to the university to resume face-to-face classes. Sara reflected on this, "So, if university will start in August, I might not return next semester, not only because I can't go back to America [due to travel restrictions] but also because I don't want to lose the safety. This is biggest question now for me...".

\section{The Salience of the English Language}

The salience of the English language, the desire to learn and speak it, and the subsequent disappointment due to the challenges posed by COVID-19 were significant themes across the majority of the participants. Several participants expressed their expectations of becoming proficient in the language by studying at a U.S. university. Sky reported:

My expectation is first to improve the English ability. I do think My English skills improved like I want to improve my vocabulary actually. I think for the 
before January and my vocabulary didn't improve it just because I feel more confident than I have been in practice with native speakers so and that makes me feel more comfortable to talk with other American students.

Some participants also mentioned their desire to improve their spoken language skills to be able to socialize better in order to fully experience the American culture. Tracy lamented:

because my English is not good, sometimes, it's very difficult to socializing and learning. So... and it is very difficult to experience real American culture.

However, these expectations were met with the challenges brought by COVID-19 and the subsequent isolation and limited human contact. Many participants reported how the social and physical isolation negatively impacted their relationship with professors. Nancy reported:

I think for me, during the quarantine time is kind of not that easy to reach out to some of the professor because they don't want to use Zoom for kind of personal question or something. They just prefer using email, but it's kind of difficult for me sometimes to arrange all things in one email because my English is not that good, but it doesn't generally any problem...it's just kind of the difficult.

Challenges associated with COVID-19 were seemingly responsible for deteriorating language skills for participants that they acquired since their arrival in America. Tao explained:

For me the biggest problem this My English language from the start of this visiting period my English was improving quickly but the last three months I guess my English go back to the same before I came to America.

These unexpected changes also caused some students to feel less confident and more concerned about their academic standing. Ruth reported:

I think the fact that before the pandemic my biggest challenge is my probably my oral communication, I still worry about my oral communication 'cause I know if I go to academic class I had to listen to teacher, what the teacher said I study by myself like before and use the book I want to more information from professors so this is my big challenge.

In addition to struggling with professional relationships with professors and deteriorating academic performance, participants also highlighted how the pandemic has negatively affected their social relationships and friendships. Michelle said:

I can feel that not all students want to talk to me or treat me very well because...maybe I would think that I'm not fluent in English speaking but the more I think ..."well, it's coronavirus 19".

In closing, Ruth's statement aptly sheds light on the concerns and lack of confidence associated with being a non-English speaking international student in a U.S. university: 
I think sometimes I don't have confidence with my English I worry about maybe I used the wrong words or vocabulary or they misunderstood sometimes.

\section{Intersectionality of Policies and Complex Decision Making}

As previously discussed, several participants frequently reported concerns around safety and its subsequent impact on their decision making. This was especially apparent in the participants' concerns with conflicting guidelines around wearing protective gear such as masks. Furthermore, these inconsistent regulations also negatively impacted participants' and their family's decision to either remain in the US or return to China. Evidently, Sky reported:

American people do not like well [sic] masks and then they do not take seriously about the COVID-19 like the Chinese people do so like my family is really worried about me so they are trying to push me like you have to go back to China now you buy your ticket now and I said the tickets really expensive at that time.

The indecision about staying in the US for in-person classes or returning to China and risking a lowered quality of education due to online classes was a consistent theme across several participants. Specifically, the concern around staying in the U.S. in order to continue in-person classes was chiefly a product of lack of information regarding safety precautions taken by the university to ensure the health and safety of all its students. Relatedly, Elva reported:

...I suppose to do to come back to America because school is gonna start and April 17 and then we have to start the next semester in person which coronavirus didn't fix this didn't make it go away and then it was still gonna have class so I really very worried about that.

These issues were further exacerbated by the travel ban imposed by the U.S. and China. Students who wished to return to China, where the situation was reportedly better, were unable to do so due to the travel ban in both countries as a way to contain the novel Coronavirus. Xin reported:

Mainly due to the travel restrictions because the travel ban and growing epidemic make my parents, myself included, worried about whether it is safe to have in-person classes next semester with around 30,000 new cases in the U.S. every day and not everyone wears the mask and easy to get infected without mask and any prevention I understand it's each person's choice whether they want to wear a mask or not.

In addition, Sky discussed the cost prohibitive air travel and the ways in which her education might be affected if the travel bans in both countries continue to be in effect. She reported:

...my family is really worried about me so they are trying to push me like you have to go back to China now, you buy your ticket now and I said the tickets' 
really expensive at that time...I think that is too expensive cause I still have to do my class and if I have to go back to China, I will have time differences and so I will take classes around 4:00 AM in the morning in China and why I should do that so I think like after the spring semester and yeah so like in March, I got a lot of stress from my family members that they pushed me to go back to China.

Similarly, visa issues have presented as significant concerns around traveling outside the U.S. Emma, who wanted to visit her chronically sick parent in Canada, was unable to do so because of administrative difficulties at the university's international student services office, thus restricting her travel plans. She reported:

So, it was very disconcerting to me that you know if something should happen to one of my parents, God forbid, with their current illness and if an emergency occurred, would I be able to fly back and if I were to fly back, would I be able to return to the U.S.

Emma further explained how she made concerted efforts to find alternatives to visit her parents by contacting embassies and border agents, but unfortunately, received mixed messages, thus adding to her helplessness and confusion.

Is education considered an "exception", essential work and according to....I contacted multiple embassies about this...according to both embassies, education is considered essential travel however, when I did contact the border directly, at least the land crossing because I was thinking it's irresponsible to fly, I could drive up, I have a car. The border agent actually could not confirm that in fact, essential travel included education. So, without knowing that for sure I didn't; wanna make the 12-hour trip home. So, it's very confusing because we're receiving many different messages which are conflicting.

These issues with visas are not only restricted to visiting families stationed outside the U.S. Participants also reported how COVID-19 is affecting their ability to secure employment in the US. Michelle reported:

For me, I think the most challenging is to get attention. We really need help with visas and jobs. So, because now, this kind of situation a lot of international students, they graduated but the school cannot give them jobs or some kind company rejects their application, so I think it's not very good for the students.

\section{Unexpected Support and Benefits}

The majority of participants reported that university professors and classmates were more intentional in communicating and checking in on them (support came more from those who worked directly with students as well as those in administration). Alice described her interactions with professors during the pandemic:

...there's not this distance between me and my professors I feel like we like Friends and I think the pandemic makes you feel like families because all 
the professors had taught me the they sent me emails and checking on me during the pandemic so they make me feel like out there my parents more like my parents.

Support went beyond the classroom as professors and administrators reached out to students who were experiencing significant challenges as described by Catherine:

I was new to an Airbnb for two weeks for self-isolating it, just my parents kind of worry about what if I'm not exposed. And then, um, my, my roommate got COVID-19, when you're not sure and she can't do the test. So, uh, during that time period, I was sharing this, um, cause I was also preparing my thesis project for, um, for my studio class. And then I was sharing this with my professor and, um, she and our Dean was like, um, trying to help me. We have a lot of conversation during that time period when I was selfisolating outside. They were trying to help me really.

Although participants did not explicitly name xenophobia, their experiences with others reaching out to them appeared to be an acknowledgement from the university community of the awareness that this was being directed toward Chinese/Asian international students in the United States. Emma's experience is an example of this:

...as for my U.S. friends, I would say, they've been very supportive. I'd say my class in general, in school, is quite liberal and progressive and so a lot of people have posted online or reached out to me...not only about coronavirus, and "hey, are you doing okay?", but specifically, knowing that I am Asian and I look Asian and so I might face discrimination while I'm literally out and about, you know, grocery shopping and all that, which has happened unfortunately, but it's comforting to know that people who do know me and care about me as an individual, do think about these things, and think about it as more than just coronavirus affecting everybody.

Interactions online and via Zoom sessions provided socialization support to many of the participants who reported feeling isolated and feeling self-conscious/ under confident in being proficient with spoken English decreased as more written than spoken English was utilized. Meihan reported the importance of using technology to connect with people during quarantine:

Well, I think it great experience for me. I think I get more attention from more people because usually very busy with, we don't have the time to take our phones and check with friends but now, all I can say is thanks to my phone and social media, without which I would have been disappointed and very sad during my situation, whereas now I'm very happy.

Technology included social media but also changes in class formats from in-person class meetings to online class meetings that was experienced positively by Tracy:

....since VCU changed to online course, I think I experienced another way of class.... and this time, the classmates are more active. We celebrated my 
classmate's birthday, together online... and [unintelligible]...we come back to each other more during this period, we encouraged each other.

\section{Discussion}

In general, international students or visiting scholars in the U.S. have the opportunities to expose firsthand home culture and American cultures and values, which could potentially benefit them academically, culturally, and socially. At the same time, international students also make invaluable contributions to the diverse American cultures by bridging global views with the local cultures through firsthand classroom experience and community-engaged activities (Bilecen, 2020). Unfortunately, the COVID-19 pandemic interrupted these interactions unexpectedly because the "flow" was forcefully paused. The pandemic has affected all international students' mobility, particularly Chinese international students who make up the largest portion of this demographic in the U.S. However, the U.S. will continue hosting a large number of international students during the post-pandemic period because the demand for international education will continue to exist, even though the enrollment might decrease in the next several years due to factors such as uncertainty and safety (Bilecen, 2020). Therefore, it is urgent for U.S. universities to address these factors by establishing a culturally responsive system to support Chinese international students regarding their specific concerns and needs.

\section{Safety Concerns}

The findings of Chinese international students' safety concerns align with other studies related to COVID-19. For example, Ma and Miller (2020) found that many international Chinese students living overseas are finding themselves faced with a double bind situation as a consequence of receiving conflicting messages from family, friends, and those in their immediate social setting. This project provides additional information about their safety concerns from personal stories and experiences during the initial COVID-19 quarantine period. Their safety concerns about the university campus and the larger community urge us to be more sensitive of international students' challenges and struggles, particularly during the pandemic. Findings of this study will help the university establish and strengthen a culturally and linguistically responsive support system that is built upon the existing resources, not only for Chinese international students, but all students on campus.

\section{The Salience of the English Language}

Findings on participants' desire to learn the English language or the anxiety of not being able to improve their spoken English language skills reflect a larger issue beyond the language proficiency, but are consistent with the existing literature on linguistic imperialism which is described as the global acquisition of the English language (Phillipson, 1992). Linguistic imperialism occurs when the native 
language of one group is dominated by another language to the extent where the people believe they must speak the foreign language to access education, participate in governance, or belong with the social elite (Phillipson, 1992). Galtung (1980) categorized imperialism in six areas- economic, political, military, communicational, cultural, and social. Appadurai (1996) explored the cultural effects of globalization through patterns of English language acquisition, discourse on multiculturalism, and ethnic violence. More than one participant expressed their concerns about their English language proficiency, particularly how that could affect their interactions with American peers and professors. This finding supports Appadurai's exploration of cultural effects of globalization, particularly through patterns of English language acquisition. Appadurai's framework for conceptualizing globalization centers on global flows that influence the spread of the English language. He described the boundaries between how one's perception of the world influences self-understanding and roles in social institutions, both within and between nations. He stated that the media's international representation of popular culture and lifestyle affects people's values, beliefs, and perceptions of their world. Some participants worried that their "English was not good enough" which further confirms Appadurai's argument on cultural effects of globalization through patterns of English. In other words, these students' perception that their "English was not very good" implies the influence of the dominating or popular culture on their perceived images.

\section{Intersectionality of Policies and Complex Decision Making}

The findings on intersectionality of policies and complex decision making reflect the reality of many international students who live between the two worlds: their home culture and the American culture, which could either complement or contradict each other and sometimes they complement and contradict each other simultaneously. When international students live between the two worlds, their decision making is often affected by policy changes related to their immigration status and academic needs, as well as their "push out" (home country) and "pull in" (destination country) cultures. This intersectionality is an ongoing complex issue that can create additional stresses unique to international students because the "push out" and "pull in" could change over time, along with the economic changes or political trends. McMahon (1992) explored the relationship between international student mobility with global politics, economics, and culture. She presented a conceptual framework that highlights push and pull factors to account for mobility. The push relates to the politics, economy, and culture of the international student's homecountry and the pull relates to the same factors in the destination country which encourage student mobility. Mazzarol and Soutar (2002) used the push-pull framework to examine international students' selection of destination country and host institution. The authors argued that social and economic factors in the country of origin push students to other countries for higher education. The decision to learn in a particular country, at a given school relates to pull factors. The large number of international Chinese students in the U.S. since China's Open Door economic policies points to the influences of social and economic factors in their selection of 
destination country. Until the pandemic, the "push out" and "pull in "was roughly balanced in terms of academic, economic, and cultural expectations from the home country and the destination country because international mobility was possible. Prior to the pandemic, the process of decision making was predictable because factors affecting the decision were "negotiable" or could be compromised as long as safety was not an issue. However, COVID-19 made safety a major issue and further complicated decision making due to political conflicts between the U.S. and China. Especially when these students were right in the middle of their academic studies, there was no easy solution for them to choose: going back home? They might not be able to reenter the destination country to finish their degree; staying in the U.S.? They might have to face more uncertain policy issues regarding their immigration status, not to mention the pressures from their home country. In other words, they were stuck.

\section{Unexpected Support and Benefits}

Educators and researchers, students and parents are increasingly concerned about what the world will look like after the pandemic. Indeed, in the short term, the number of international student enrollment may drop significantly in the following years due to travel restrictions, political conflicts, and economic stresses. In the long run, however, international student mobility of higher education will not stagnate, because the foundation of international education is "talent training" (Wei, 2020) and the higher education community in each country provides an ideal context for talent training with innovation and scientific inquiry. Results of this study point to this direction, despite all the challenges identified. This finding also aligns with the survey results from Chi et al.'s study (2020) that more than half of the surveyed Chinese college students showed strong intrinsic motivation for positive growth during the COVID-19 pandemic. The majority of the participants in the current study also expressed positive attitudes towards their peers and professors, confirming the importance and value of international education.

Fortunately, the university community in each country has a tradition for promoting academic and cultural exchanges and our findings on unexpected support and benefits of the current study confirm this positive climate with students' perceptions and experiences during the quarantine period. Even during the pandemic age, universities from both China and the U.S. have taken proactive approaches to supporting international students. As stated by Yang Xinyu, minister counselor for educational affairs at the Chinese Embassy in the U.S., "The U.S. and Chinese educational communities have come out to support each other as unfavorable U.S. policies and the surging COVID-19 pandemic disrupted the educational exchanges between the two countries (Cheng, 2020)." For example, when over 200 Chinese students at Delaware University could not return to the U.S. in February 2020 for the spring semester due to the U.S. travel ban, the university worked collaboratively with the China Embassy's education affairs office to make sure that the students could proceed with their studies in a flexible schedule. Another example is that many universities from both China and the U.S. wrote to their partner universities to offer support during 
the pandemic and to reassure with an even stronger interest and confidence of collaboration after the pandemic (Cheng, 2020). Additionally, the Emergency Student Fund of the Institute of International Education responded timely to COVID-19 by providing grants to international students on U.S. campuses with financial hardships.

The findings of the current study further confirm the need for establishing an ongoing support system within and between higher education institutions to support international students. Having a culturally and linguistically responsive system is the key to promoting the success of all international students in the world and cultivating talent training through innovative international exchanges academically, culturally, and socially. There is no doubt that international students will continue to account for a significant portion of all students in the U.S. higher education.

\section{Limitations}

Although Zoom provides convenience and flexibility to conduct the interviews, we consider it as a limitation of the current study, because we suspect that not all participants were comfortable expressing their responses or concerns in front of other participants during the focus group discussions. Future research may use individual interviews to gather in-depth perceptions on sensitive topics such as race, religion, or political orientations. Another limitation of the study was that we only focused on Chinese international students from one major university in the U.S. Although our target university is a public urban institution including students from diverse backgrounds, Chinese international students from other universities may bring different and valuable perspectives in terms of their lived experiences in the U.S. Consequently, future research can expand the sampling by including Chinese international students from other universities in the U.S.

\section{Recommendations for Future Research}

Findings of the current study suggest that racism could be an ongoing issue, despite that no explicit statement was expressed by any participants. From their stories and struggles related to racial biases, we realized that unconscious racism might be experienced by Chinese international students in the university community as well as the larger society, although they never used the term "racism." Future research can focus on racial issues through an exploration of the intersectionality of racism, education, and culture within the pre- and post-pandemic context. Further investigation of the long-term impact of COVID-19 on international students is needed. We employed a qualitative research method to explore Chinese international students' lived experience, which provides meaningful and timely information about their challenges and struggles. Findings of the current study help us understand and explain participants' experiences from multiple perspectives, which point future researchers to further investigate the complex intersectional phenomena. Examining individuals and their perspectives at the intersectionality of multiple factors is a research paradigm that requires a mixed-method research design for inquiry because the complexity of intersectionality demands multiple forms of data sources and analysis (Griffin \& 
Museus, 2011). Within the context of intersectionality research, mixed methods can help us understand complex phenomena representing individual as well as group perspectives (Creswell \& Creswell, 2017). In order to better support the 400,000 international Chinese students in the U.S., researchers need to develop a mixedmethods design to explore international students' experiences by involving more universities across the state and the nation. Both qualitative and quantitative data are needed to identify issues and to generalize the findings to benefit all international students from other countries, in addition to those from China.

Findings of our current study helps provide valuable information for establishing an ongoing support system to help international students' transition from their home country to the destination country. Future research can examine the effectiveness of these support systems on international students' academic success, mental health, and overall well-being.

\section{Conclusion}

In this study we explored Chinese international students' lived experiences in the U.S during the initial COVID-19 quarantine period. The COVID-19 pandemic has affected everyone's life in multiple ways. A survey of over 700 colleges indicated the total number of international students in the U.S. decreased by $16 \%$ in the fall 2020 while enrollments of new international students decreased by $43 \%$ (Open Doors Report, 2020). While experts predict that when the pandemic ends, students who have deferred admission or application would want to come, the need to serve these students to ensure their success could vary from person to person, based on their experiences during the COVID-19 period. Findings of the current study may help future international students in dealing with crises or coping with stresses, expectedly or unexpectedly. Some of the impacts could last long after the pandemic ends and there is a need to address these to establish normalcy. Even with the decline of international student enrollment, Chinese international students in the U.S. still account for the largest portion (about 35\%) among all international students (Redden, 2020). Therefore, universities in the U.S need to be prepared with adequate resources and support to help prospective international students succeed when they do choose to come back to the U.S. or choose the U.S. as their destination for academic pursuits.

\section{Declarations}

Conflict of Interest The authors declare that there is no conflict of interest. 


\section{References}

Amour, M. (2020). China Offers Help to Students in U.S., for a Price. Inside Higher Ed. Retrieved from https://www.insidehighered.com/news/2020/04/07/china-offers-help-students-us-price. Accessed 20 Apr 2020

Appadurai, A. (1996). Modernity at large: Cultural dimensions of globalization. University of Minnesota Press.

Bilecen, B. (2020). Commentary: COVID-19 pandemic and higher education: International mobility and students' social protection. International Migration, 58(4), 263-266. https://doi.org/10.1111/imig. 12749

Cheng, Z. (2020). U.S. Chinese educational communities' mutual support "silver lining" amid pandemic: Senior diplomat. Available: http://www.ecns.cn/news/2020-12-13/detail-ihaetmvy3495797.shtml. Accessed 15 Dec 2020

Chi, X., Becker, B., Yu, Q., Willeit, P., Jiao, C., Huang, L., et al. (2020). Prevalence and psychosocial correlates of mental health outcomes among Chinese college students during the coronavirus disease (COVID-19) pandemic. Frontiers in Psychiatry, 11, 803. https://doi.org/10.3389/fpsyt.2020. 00803

Creswell, J. W., \& Creswell, J. D. (2017). Research design: Qualitative, quantitative, and mixed methods approaches. Sage.

Downing, M., \& Cooney, A. (2012). Research approaches related to phenomenology: Negotiating a complex landscape. Journal of Nursing Research, 20(2), 21-27. https://doi.org/10.7748/nr2012.11.20.2. 21.c9440

Elo, S., Kaariainen, M., Kanste, O., et al. (2014). Qualitative content analysis: A focus on trustworthiness. SAGE Open, 4(1), 1-10.

Galtung, J. (1980). The changing interface between peace and development in a changing world. Bulletin of Peace Proposals, 11(2), 145-149.

Giorgi, A., Giorgi, B., \& Morley, J. (2017). The descriptive phenomenological psychological method. In Willig \& Rogers (Eds.), The Sage handbook of qualitative in psychology (2nd ed., pp. 176-192). Publisher: Sage.

Griffin, K. A., \& Museus, S. D. (2011). Application of mixed-methods approaches to higher education and intersectional analyses. New Directions for Institutional Research, 2011(151), 15-26.

Henry, J., \& Bensimon, O. (2020). Victim of possible corona-virus hate crime in Queens speaks out. https://www.nypost.com/2020/03/14/victim-of-possible-coronavirus-hate-crime-in-queens-speaksout. Accessed 20 Apr 2020

Holloway, I., \& Todres, L. (2003). The status of method: Flexibility, consistency and coherence. Qualitative Research, 3, 345-357

Lincoln, Y. S., \& Guba, E. G. (1985). Naturalistic inquiry. Sage Publications.

Litam, S. D. A. (2020) “Take Your Jung Flu Back to Wuhan”. Counseling Asians, Asian Americans, and Pacific Islanders with Race-Based Trauma Related to COVID-19. Professional Counselor, 10(2), 1440156.

Ma, H., \& Miller, C. (2020). Trapped in a double bind: Chinese overseas student anxiety during the COVID-19 pandemic. Health Communication. https://doi.org/10.1080/10410236.2020.1775439

Mazzarol, T., \& Soutar, G. N. (2002). "push-pull" factors influencing international student destination choice. The International Journal of Educational Management, 16(2), 82-90.

McMahon, M. (1992). Higher education in a world market. Higher Education, 24(4), 465-482.

Moustakas, C. E. (1994). Phenomenological research methods. Sage Publications, Inc.

Open Doors Report (2020). United States hosts over 1 million international students for the fifth consecutive year. Available: https://www.iie.org/en/Why-IIE/Announcements/2020/11/2020-Open-DoorsReport. Accessed 15 Dec 2020

Palmer, E. (2020). Asian woman allegedly attacked in New York subway station for wearing protective mask. Newsweek. https://www.newsweek.com/new-york-subway-attack-coronavirus-woman-mask1485842. Accessed 20 Apr 2020

Phillipson, R. (1992). Linguistic imperialism. Oxford University Press.

Redden, E. (2020). International student numbers decline. Available: https://www.insidehighered.com/ news/2020/11/16/survey-new-international-enrollments-drop-43-percent-fall?utm_source=Inside+ Higher+Ed\&utm_campaign=0384e91223-DNU_2020_COPY_02\&utm_medium=email\&utm_ 
term=0_1fcbc04421-0384e91223-236613946\&mc_cid=0384e91223\&mc_eid=30d4263bba. Accessed 15 Dec 2020

Roberto, K. J., Johnson, A. F., \& Rauhaus, B. M. (2020). Stigmatization and prejudice during the COVID-19 pandemic. Administrative Theory \& Praxis, 42(3), 364-378.

Schild, L., Chen, L., Blackburn, J., Stringhini, G., Zhang, Y., \& Zannettou, S. (2020). "Go eat a bat, Chang!": An early look on the emergence of Sinophobic behavior on web communities in the face of COVID-19. Available: https://arxiv.org/abs/2004.04046. Accessed 20 Apr 2020

Tavernise, S., \& Oppel, R. A. (2020). Spit on, yelled at, attacked: Chinese-Americans fear for their safety. The New York Times. https://www.nytimes.com/2020/03/23/us/chinese-coronavirus-racist-attacks. html. Accessed 20 Apr 2020

Tobin, G., \& Begley, C. (2004). Methodological rigour within a qualitative framework. Journal of Advanced Nursing, 48(4), 388-396.

Wang, T. (2020). The COVID-19 crisis and cross-cultural experience of China's international students: A possible generation of glocalized citizens? ECNU Review of Education, 1-6. https://doi.org/10. $1177 / 2096531120931519$

Wei, L. (2020). International education strategy seminar under the challenge of COVID-19. The Study Board Service Branch of China Education International Exchange Association

Yu, M., Tian, F., Cui, Q., \& Wu, H. (2021). BMC Psychiatry 21:66. https://doi.org/10.1186/ s12888-021-03066-9

Publisher's Note Springer Nature remains neutral with regard to jurisdictional claims in published maps and institutional affiliations. 